\title{
Multivariate Extremes of Generalized Skew-normal Distributions *
}

\author{
Natalia Lysenko $^{\mathrm{a}, *}$, Parthanil Roy ${ }^{\mathrm{b}}$, Rolf Waeber ${ }^{\mathrm{a}, 1}$ \\ ${ }^{a}$ ETH Zurich, Department of Mathematics, 8092 Zurich, Switzerland \\ ${ }^{\mathrm{b}}$ ETH Zurich, Department of Mathematics, 8092 Zurich, Switzerland and \\ Department of Statistics and Probability, Michigan State University, East Lansing, \\ MI 48824, USA
}

\begin{abstract}
We explore extremal properties of a family of skewed distributions extended from the multivariate normal distribution by introducing a skewing function $\pi$. We give sufficient conditions on the skewing function for the pairwise asymptotic independence to hold. We apply our results to a special case of the bivariate skew-normal distribution and finally support our conclusions by a simulation study which indicates that the rate of convergence is quite slow.
\end{abstract}

Key words: Extreme values, multivariate extremes, domain of attraction, asymptotic independence, generalized skew-normal, skewing function

\section{Introduction}

Understanding stochastic properties of multivariate extremes is essential in many applied fields. Applications of extreme value theory in the insurance and banking sectors are discussed, for instance, in Embrechts et al. (1997), McNeil et al. (2005), Balkema and Embrechts (2007). The Gaussian distribution lies at the heart of many models in finance and insurance. However, real data on insurance losses and financial returns often indicate departures from

\footnotetext{
ऋ Supported by the RiskLab of the Department of Mathematics, ETH Zurich

* Corresponding author

Email address: natalia.lysenko@math.ethz.ch (Natalia Lysenko).

1 Present address: School of Operations Research and Information Engineering, Cornell University, Ithaca, NY 14853, USA
} 
normality such as the presence of skewness (see, e.g., Lane (2000)), which makes mathematical convenience gained by assuming normality unjustified.

Although multivariate extremes have been studied in detail for many standard distributions, it is in general not known what happens if we relax one or more of the 'nice' properties of such distributions. The goal of this paper is to explore the extremal behavior of the multivariate generalized skew-normal distributions (see Section 2 and also Genton (2004) for a more detailed discussion) obtained from the multivariate normal distribution by relaxing the property of elliptical symmetry using a so-called skewing function. An important role played by skewed distributions in many fields including finance and insurance, biology, meteorology, astronomy, etc. (cf. Hill and Dixon (1982), Azzalini and Capitanio (1999), Genton (2004)) motivates our interest in extremal properties of this class of distributions.

The paper is organized as follows. In Section 2 we give the definition of the multivariate generalized skew-normal distributions and review the results of Chang and Genton (2007) on the extremal behavior of such distributions in the univariate case. In Section 3 we state sufficient conditions for asymptotic independence in the multivariate set-up along with some examples. Finally, in Section 4. we investigate the rate of convergence to the extreme value distribution for both univariate and multivariate skew-normal distributions using simulations. Throughout the paper, we use the following common abbreviations: $c d f$ for cumulative distribution function, $p d f$ for probability density function, i.i.d. for independent and identically distributed.

\section{Preliminaries}

In this section we define the class of generalized skew-normal distributions and review its extremal properties in the univariate case.

Definition 2.1 A $d$-dimensional random vector $\mathbf{X}=\left(X_{1}, \ldots, X_{d}\right)^{T}$ follows a generalized skew-normal (GSN) distribution with location parameter $\boldsymbol{\xi}$, scale parameter $\Omega=\left(\omega_{i j}\right)$ and skewing function $\pi$, denoted by $\mathbf{X} \sim G S N_{d}(\boldsymbol{\xi}, \Omega, \pi)$, if its density function is given by

$$
g(\mathbf{x})=2 \phi_{d}(\mathbf{x} ; \boldsymbol{\xi}, \Omega) \pi(\mathbf{x}-\boldsymbol{\xi}),
$$

where $\pi: \mathbb{R}^{d} \rightarrow[0,1]$ satisfies $\pi(-\mathbf{x})=1-\pi(\mathbf{x})$ and $\phi_{d}(\mathbf{x} ; \boldsymbol{\xi}, \Omega)$ is the pdf of a $d$-dimensional normal random vector with mean vector $\boldsymbol{\xi}$ and covariance matrix $\Omega$.

It is not difficult to check that $g$ is a valid pdf for any skewing function $\pi$. One of the nice properties of this class of distributions is that the marginals also 
belong to the same class; see Proposition 2.3 The following example gives an important special case.

Example 2.2 (Multivariate flexible skew-normal distribution) If $\pi(\mathrm{x})$ $=H\left(P_{K}(\mathbf{x})\right)$, where $H$ is any cdf of a continuous random variable symmetric around 0 and $P_{K}$ is an odd polynomial of order $K$ defined on $\mathbb{R}^{d}$, then $\mathbf{X}$ is said to follow a flexible skew-normal distribution, which has the density of the form $g(\mathbf{x})=2 \phi_{d}(\mathbf{x} ; \boldsymbol{\xi}, \Omega) H\left(P_{K}(\mathbf{x})\right)$; see Fig. 1 for an example. This class of distributions, introduced by $\mathrm{Ma}$ and Genton (2004), can systematically model light tails, multimodality and skewness. If we take $K=1, H=\Phi$ (the stan-
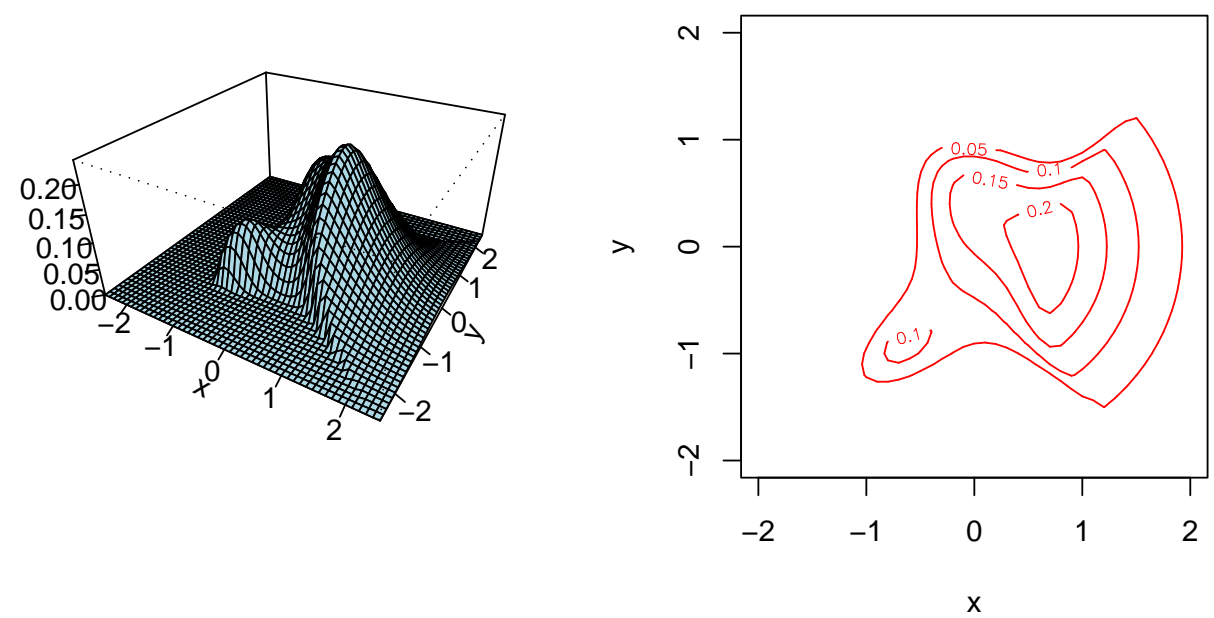

Fig. 1. The density and contours of a bivariate flexible skew-normal distribution with $\boldsymbol{\xi}=\mathbf{0}, \Omega=I_{2}, H=\Phi, K=3$, and $P_{K}(x, y)=x+y-4 x^{2} y-2 x y^{2}+2 x^{3}-y^{3}$.

dard normal cdf) and $P_{K}(\mathbf{x})=\boldsymbol{\alpha}^{T} \mathbf{x}$ for some $\boldsymbol{\alpha} \in \mathbb{R}^{d}$ so that the density has a form $g(\mathbf{x})=2 \phi_{d}(\mathbf{x} ; \boldsymbol{\xi}, \Omega) \Phi\left(\boldsymbol{\alpha}^{T} \mathbf{x}\right)$ then this special case is referred to as multivariate skew-normal distribution with location parameter $\boldsymbol{\xi}$, scale parameter $\Omega$ and shape parameter $\boldsymbol{\alpha}$ (denoted as $\mathrm{X} \sim S N_{d}(\boldsymbol{\xi}, \Omega, \boldsymbol{\alpha})$ ). This distribution, introduced by Azzalini and Dalla Valle (1996), has the advantage that it is still mathematically tractable as well as able to model various unimodal but non-elliptical situations. Clearly, for $\boldsymbol{\alpha}=\mathbf{0}$ it is simply the $d$-dimensional normal distribution, $N_{d}(\boldsymbol{\xi}, \Omega)$.

The following proposition shows that the marginals of the GSN distributions are also GSN.

Proposition 2.3 Suppose $\mathbf{X} \sim G S N_{d}(\boldsymbol{\xi}, \Omega, \pi)$ and $\mathbf{X}$ is partitioned as $\mathbf{X}^{T}=$ 
$\left(\mathbf{X}_{(1)}^{T}, \mathbf{X}_{(2)}^{T}\right)$ of dimensions $h$ and $d-h$, respectively; denote by

$$
\Omega=\left(\begin{array}{ll}
\Omega_{11} & \Omega_{12} \\
\Omega_{21} & \Omega_{22}
\end{array}\right) \text { and } \boldsymbol{\xi}=\left(\begin{array}{l}
\boldsymbol{\xi}_{1} \\
\boldsymbol{\xi}_{2}
\end{array}\right)
$$

the corresponding partitions of $\Omega$ and $\boldsymbol{\xi}$, respectively. Then the marginal distribution of $\mathbf{X}_{(1)}$ is $G S N_{h}\left(\boldsymbol{\xi}_{1}, \Omega_{11}, \pi^{(1)}\right)$ with $\pi^{(1)}(\boldsymbol{y})=E\left(\pi(\boldsymbol{Z}-\boldsymbol{\xi}) \mid \boldsymbol{Z}^{(1)}=\boldsymbol{y}\right)$, where $\boldsymbol{Z} \sim N_{d}(\boldsymbol{\xi}, \Omega)$ and $\boldsymbol{Z}=\left(\boldsymbol{Z}^{(1)}, \boldsymbol{Z}^{(2)}\right)$ is the corresponding partition of $\boldsymbol{Z}$.

Proof Since $\mathbf{X} \sim G S N_{d}(\boldsymbol{\xi}, \Omega, \pi)$, it has density $g(\mathbf{x})=2 \phi_{d}(\mathbf{x} ; \boldsymbol{\xi}, \Omega) \pi(\mathbf{x}-\boldsymbol{\xi})$ with notations in Definition 2.1. Let $\mathbf{x}^{T}=\left(\mathbf{y}^{T}, \mathbf{z}^{T}\right)$ be the corresponding partition of the variable $\mathbf{x}$. Then the density of $\mathbf{X}^{(1)}$ is given by (with the vectors of variables written as row vectors instead of column vectors)

$$
\begin{aligned}
h\left(\mathbf{y}^{T}\right) & =\int_{\mathbb{R}^{d-h}} g\left(\mathbf{y}^{T}, \mathbf{z}^{T}\right) d \mathbf{z} \\
& =2 \int_{\mathbb{R}^{d-h}} \phi_{d}\left(\mathbf{y}^{T}, \mathbf{z}^{T} ; \boldsymbol{\xi}, \Omega\right) \pi\left(\left(\mathbf{y}-\boldsymbol{\xi}^{(1)}\right)^{T},\left(\mathbf{z}-\boldsymbol{\xi}^{(2)}\right)^{T}\right) d \mathbf{z} \\
& =2 \phi_{h}\left(\mathbf{y}^{T} ; \boldsymbol{\xi}^{(\mathbf{1})}, \Omega_{11}\right) \int_{\mathbb{R}^{d-h}} \pi\left(\left(\mathbf{y}-\boldsymbol{\xi}^{(1)}\right)^{T},\left(\mathbf{z}-\boldsymbol{\xi}^{(2)}\right)^{T}\right) \psi\left(\boldsymbol{z}^{T}\right) d \mathbf{z}
\end{aligned}
$$

where $\psi$ is the density of $\boldsymbol{Z}^{(2)}$ given $\boldsymbol{Z}^{(1)}=\boldsymbol{y}$. This proves the result.

We now turn our attention to the extremal behavior of these distributions in the univariate case. Without loss of generality it can be assumed that the location parameter is 0 and the scale parameter is 1 . We use the notation $X \sim G S N(\pi)$ and $X \sim S N(\alpha)$ to mean $X \sim G S N_{1}(0,1, \pi)$ and $X \sim$ $S N_{1}(0,1, \alpha)$, respectively. The following result summarizes the implications of Propositions 2.1 and 2.2 in Chang and Genton (2007) for univariate generalized skew-normal distributions. For the underlying extreme value theory, see, for example, Resnick (1987), Embrechts et al. (1997), de Haan and Ferreira (2006).

Proposition 2.4 (Chang and Genton (2007)) Let $F$ be the cdf of a random variable $X \sim G S N(\pi)$. Assume that the skewing function $\pi: \mathbb{R} \rightarrow[0,1]$ and the cdf $F$ satisfy the following conditions:

i) $\pi$ is continuous and there exists a constant $M>0$ such that $\pi(x)$ is positive and monotone for $x>M$;

ii) $\pi$ has continuous second derivative;

iii) there exists a constant $M^{*}>0$ such that $F^{\prime \prime}(x)<0$ for $x>M^{*}$;

$i v)$ either $\lim _{x \rightarrow \infty} \pi(x)=\eta \in(0,1]$ or $\lim _{x \rightarrow \infty} \frac{(1-F(x)) F^{\prime \prime}(x)}{\left(F^{\prime}(x)\right)^{2}}=-1$.

Then $F \in M D A(\Lambda)$, where $\Lambda$ denotes the Gumbel distribution given by $\Lambda(x)=\exp \left(-e^{-x}\right)$ for $x \in(-\infty, \infty)$. 
Here the notation $G \in M D A(\Lambda)$ means $G$ belongs to the maximum domain of attraction of $\Lambda$ (the Gumbel distribution). Using Proposition 2.4 Chang and Genton (2007) established that the univariate flexible skew-normal distribution with $H=\Phi$ (and hence in particular the univariate skew-normal distribution) belongs to the maximum domain of attraction of the Gumbel distribution.

\section{Conditions for Asymptotic Independence}

Recall that a $d$-dimensional random vector $\mathbf{X}=\left(X_{1}, \ldots, X_{d}\right)^{T}$ with cdf $F$ is said to be asymptotically independent if $F$ is in the (componentwise) maximum domain of attraction of a distribution $G$ with independent components (i.e., $G(\mathbf{x})=\prod_{i=1}^{d} G_{i}\left(x_{i}\right)$ where $G_{i}$ is the $i^{t h}$ marginal of $G$ ). Since the density $g$ in Definition 2.1] is strongly connected to the multivariate normal density and any multivariate normal random vector with pairwise correlations less than 1 is asymptotically independent (see Sibuva (1960)), we expect the asymptotic independence to hold also for a generalized skew-normal vector as long as the skewing function $\pi$ satisfies some mild conditions. As asymptotic independence is essentially a pairwise concept (see Remark 6.2.5 in de Haan and Ferreira $(2006)$ ), in the next two results we give sufficient conditions for pairwise asymptotic independence of a generalized skew-normal random vector $\mathbf{X}$ in terms of the skewing functions of the univariate and bivariate marginals, which can be calculated from the skewing function $\pi$ using Proposition 2.3.

Theorem 3.1 Consider $\mathbf{X} \sim G S N_{d}(\boldsymbol{\xi}, \Omega, \pi)$. Fix $i, j \in\{1,2, \ldots, d\}$ with $i \neq$ $j$ and $\frac{\omega_{i j}}{\sqrt{\omega_{i i} \omega_{j j}}}<1$. Let $\pi_{i}, \pi_{j}: \mathbb{R} \rightarrow[0,1]$ be the skewing functions of $X_{i}$ and $X_{j}$, respectively. Assume that the skewing functions satisfy the following conditions:

i) there exists a constant $M_{1} \in \mathbb{R}$ such that either $\pi_{i}(x) \leq \pi_{j}(x)$ or $\pi_{j}(x) \leq$ $\pi_{i}(x)$ for all $x \geq M_{1}$

ii) $\liminf _{u \rightarrow \infty} \pi_{i}(u)>0, \liminf _{u \rightarrow \infty} \pi_{j}(u)>0$.

Then $X_{i}$ and $X_{j}$ are asymptotically independent.

Proof To prove this theorem we assume without loss of generality that $\xi_{k}=0$ and $\omega_{k k}=1$ for all $k=1, \ldots, d$. Furthermore, for simplicity of notation, we only consider the case $i=1, j=2$, and $\pi_{1}(x) \leq \pi_{2}(x)$ for $x$ larger than some constant $M_{1}$. Define $\omega:=\omega_{12}<1$. By Theorem 6.2.3 in de Haan and Ferreira (2006), in order to establish asymptotic independence of $X_{1}$ and $X_{2}$ we need to show that

$$
\lim _{t \rightarrow \infty} \frac{P\left(X_{1}>U_{1}(t), X_{2}>U_{2}(t)\right)}{P\left(X_{1}>U_{1}(t)\right)}=0
$$


where $U_{i}(t):=\inf \left\{x \in \mathbb{R}: F_{i}(x) \geq 1-1 / t\right\}$, for $i=1$, 2. Since we assumed that $\pi_{1}(x) \leq \pi_{2}(x)$ for large $x$, it follows that $U_{1}(t) \leq U_{2}(t)$ for large $t$. Note that by condition $i i) \pi_{1}(u)>0$ for large $u$ and therefore $U_{1}(t) \rightarrow \infty$ as $t \rightarrow \infty$. So the limit in (3.1) can be bounded above as follows

$$
\begin{aligned}
\lim _{t \rightarrow \infty} \frac{P\left(X_{1}>U_{1}(t), X_{2}>U_{2}(t)\right)}{P\left(X_{1}>U_{1}(t)\right)} & \leq \lim _{t \rightarrow \infty} \frac{P\left(X_{1}>U_{1}(t), X_{2}>U_{1}(t)\right)}{P\left(X_{1}>U_{1}(t)\right)} \\
& =\lim _{u \rightarrow \infty} \frac{P\left(X_{1}>u, X_{2}>u\right)}{P\left(X_{1}>u\right)}
\end{aligned}
$$

Hence, it is enough to show that the limit in (3.2) is equal to zero. From condition $i i)$, there exist constants $M_{2}, c_{0}>0$ such that $\pi_{1}(u)>c_{0}$ for all $u>M_{2}$. Hence, for all $u>M_{2}$, the denominator in (3.2) can be bounded below by

$$
P\left(X_{1}>u\right)=\int_{u}^{\infty} 2 \phi(x) \pi_{1}(x) d x \geq 2 c_{0} \int_{u}^{\infty} \phi(x) d x
$$

and the numerator of (3.2) can be bounded above by

$$
\begin{aligned}
P\left(X_{1}>u, X_{2}>u\right) & =\int_{u}^{\infty} \int_{u}^{\infty} 2 \phi_{2}(x, y ; \omega) \pi_{12}(x, y) d x d y \\
& \leq 2 \int_{u}^{\infty} \int_{u}^{\infty} \phi_{2}(x, y ; \omega) d x d y
\end{aligned}
$$

where $\pi_{12}: \mathbb{R}^{2} \rightarrow[0,1]$ is the bivariate skewing function of $\left(X_{1}, X_{2}\right)$.

Suppose $\left(Z_{1}, Z_{2}\right) \sim N_{2}\left(\mathbf{0}, \Sigma_{\omega}\right)$ with $\Sigma_{\omega}:=\left(\begin{array}{cc}1 & \omega \\ \omega & 1\end{array}\right)$. Combining the above bounds, we get

$$
\begin{aligned}
\frac{P\left(X_{1}>u, X_{2}>u\right)}{P\left(X_{1}>u\right)} & \leq \frac{\int_{u}^{\infty} \int_{u}^{\infty} \phi_{2}(x, y ; \omega) d x d y}{c_{0} \int_{u}^{\infty} \phi(x) d x} \\
& =\frac{1}{c_{0}} \frac{P\left(Z_{1}>u, Z_{2}>u\right)}{P\left(Z_{1}>u\right)} \rightarrow 0
\end{aligned}
$$

as $u \rightarrow \infty$ by Corollary 5.28 in Resnick (1987). This completes the proof.

The following corollary is an immediate consequence of Proposition 2.4 and Theorem 3.1 .

Corollary 3.2 Let $F$ be the cdf of a bivariate generalized skew-normal random vector $\mathbf{X} \sim G S N_{2}(\boldsymbol{\xi}, \Omega, \pi)$ with $\frac{\omega_{12}}{\sqrt{\omega_{11} \omega_{22}}}<1$. Assume that the skewing functions $\pi_{1}$ and $\pi_{2}$ satisfy the following conditions: 
i) there exists a constant $M_{1} \in \mathbb{R}$ such that either $\pi_{1}(x) \leq \pi_{2}(x)$ or $\pi_{2}(x) \leq$ $\pi_{1}(x)$ for all $x \geq M_{1}$

ii) $\pi_{1}$ and $\pi_{2}$ are continuous and there exists a constant $M_{2}>0$ such that both $\pi_{1}(x)$ and $\pi_{2}(x)$ are monotone for $x>M_{2}$;

iii) $\pi_{1}$ and $\pi_{2}$ have continuous second derivatives;

$i v)$ there exists a constant $M^{*}>0$ such that $f_{1}^{\prime}(x)<0$ and $f_{2}^{\prime}(x)<0$ for $x>M^{*}$, where $f_{i}$ is the pdf of $X_{i}, i=1,2$;

v) $\lim _{x \rightarrow \infty} \min \left\{\pi_{1}(x), \pi_{2}(x)\right\}=\eta \in(0,1]$.

Then $F \in M D A(G)$, where

$$
G\left(x_{1}, x_{2}\right)=\exp \left(-e^{-x_{1}}-e^{-x_{2}}\right) .
$$

Condition $i$ ) on the skewing function in Theorem 3.1 is often satisfied. Since condition $i i$ ) is rather stringent, we relax it slightly by introducing other requirements on the skewing functions in the next result.

Theorem 3.3 Let $\mathbf{X} \sim G S N_{d}(\boldsymbol{\xi}, \Omega, \pi)$ as in Theorem 3.1. Fix $i, j \in\{1,2, \ldots, d\}$ with $\frac{\omega_{i j}}{\sqrt{\omega_{i i} \omega_{j j}}}<1$ and $i \neq j$. Let $\pi_{i}, \pi_{j}: \mathbb{R} \rightarrow[0,1]$ be the skewing functions of $X_{i}$ and $X_{j}$ respectively, and $\pi_{i j}: \mathbb{R}^{2} \rightarrow[0,1]$ be the bivariate skewing function of $\left(X_{i}, X_{j}\right)$. Assume that $\pi_{i}$ and $\pi_{j}$ satisfy condition $\left.i\right)$ of Theorem 3.1 and additionally

ii) there exists a constant $M_{2} \in \mathbb{R}$ such that both $\pi_{i}(x)$ and $\pi_{j}(x)$ are monotone for $x>M_{2}$;

iii) there exists a constant $M_{3} \in \mathbb{R}$ such that $\pi_{i j}(x, y)$ is monotone for $x, y>$ $M_{3}$ in the sense that either $\pi_{i j}\left(x^{\prime}, y^{\prime}\right) \leq \pi_{i j}(x, y)$ for all $x^{\prime} \geq x$ and $y^{\prime} \geq y$ or $\pi_{i j}\left(x^{\prime}, y^{\prime}\right) \geq \pi_{i j}(x, y)$ for all $x^{\prime} \geq x$ and $y^{\prime} \geq y$;

$i v)$ there exist constants $b>1, u_{0}, C>0$ such that $\pi_{i j}(u, u) \leq C \pi_{i}(b u)$ and $\pi_{i j}(u, u) \leq C \pi_{j}(b u)$ for all $u \geq u_{0}$.

Then $X_{i}$ and $X_{j}$ are asymptotically independent.

Proof As in the proof of Theorem 3.1] we assume $\xi_{k}=0$ and $\omega_{k k}=1$ for all $k=1, \ldots, d, i=1, j=2$, and $\pi_{1}(x) \leq \pi_{2}(x)$ for $x>M_{1}$. Once again it is enough to show that the limit (3.2) is equal to zero. We may also assume that $\lim _{u \rightarrow \infty} \pi_{1}(u)=0$ since otherwise we can use Theorem 3.1 Due to condition $i v)$, it follows that $\lim _{u \rightarrow \infty} \pi_{12}(u, u)=0$. This, combined with condition $\left.i i i\right)$, implies that both $\pi_{1}$ and $\pi_{12}$ are eventually decreasing functions. This gives the following upper bound on the numerator of (3.2) for large enough $u$ :

$$
\begin{aligned}
P\left(X_{1}>u, X_{2}>u\right) & \leq 2 \pi_{12}(u, u) \int_{u}^{\infty} \int_{u}^{\infty} \phi_{2}(x, y ; \omega) d x d y \\
& =2 \pi_{12}(u, u) P\left(Z_{1}>u, Z_{2}>u\right) \\
& \leq 2 \pi_{12}(u, u) P\left(Z_{1}+Z_{2}>2 u\right)
\end{aligned}
$$


with $\left(Z_{1}, Z_{2}\right) \sim N_{2}\left(\mathbf{0}, \Sigma_{\omega}\right)$ as before. Since $\pi_{1}$ is eventually decreasing, for large $u$ and $b>1, \pi_{1}(b u) \leq \pi_{1}(u)$ and hence the denominator in (3.2) can be bounded below by

$$
P\left(u<X_{1}<b u\right) \geq 2 \pi_{1}(b u) \int_{u}^{b u} \phi(x) d x=2 \pi_{1}(b u) P\left(u<Z_{1} \leq b u\right) .
$$

Using the above bounds along with condition $i v$ ), we get

$$
\begin{aligned}
\lim _{u \rightarrow \infty} \frac{P\left(X_{1}>u, X_{2}>u\right)}{P\left(X_{1}>u\right)} & \leq C \lim _{u \rightarrow \infty} \frac{P\left(Z_{1}+Z_{2}>2 u\right)}{P\left(u<Z_{1} \leq b u\right)} \\
& =C \lim _{u \rightarrow \infty} \frac{1-\Phi(a u)}{\Phi(b u)-\Phi(u)}
\end{aligned}
$$

where $a:=\sqrt{\frac{2}{1+\omega}}>1$. Applying l'Hôpital's rule, we obtain

$$
\lim _{u \rightarrow \infty} \frac{P\left(X_{1}>u, X_{2}>u\right)}{P\left(X_{1}>u\right)} \leq C \lim _{u \rightarrow \infty} \frac{a e^{-\frac{a^{2} u^{2}}{2}}}{e^{-\frac{u^{2}}{2}}-b e^{-\frac{b^{2} u^{2}}{2}}}=0
$$

since $a, b>1$. This completes the proof.

Example 3.4 (The Bivariate Skew-Normal Case) We now apply Theorem 3.1 and Theorem 3.3 to a bivariate skew-normal random vector. We use the notation $\mathbf{X}=\left(X_{1}, X_{2}\right)^{T} \sim S N_{2}\left(\alpha_{1}, \alpha_{2} ; \omega\right)$ to mean $\mathbf{X} \sim S N_{2}\left(\mathbf{0}, \Omega,\left(\alpha_{1}, \alpha_{2}\right)^{T}\right)$ with $\Omega=\left(\begin{array}{ll}1 & \omega \\ \omega & 1\end{array}\right)$. In this case, using Proposition 2 in Azzalini and Capitanio (1999), it follows that $X_{i} \sim S N_{1}\left(0,1, \bar{\alpha}_{i}\right), i=1,2$, where

$$
\bar{\alpha}_{1}=\frac{\alpha_{1}+\omega \alpha_{2}}{\sqrt{1+\left(1-\omega^{2}\right) \alpha_{2}^{2}}} \quad \text { and } \quad \bar{\alpha}_{2}=\frac{\alpha_{2}+\omega \alpha_{1}}{\sqrt{1+\left(1-\omega^{2}\right) \alpha_{1}^{2}}} ;
$$

i.e., $\pi_{i}(x)=\Phi\left(\bar{\alpha}_{i} x\right), i=1,2$, and $\pi_{12}\left(x_{1}, x_{2}\right)=\Phi\left(\alpha_{1} x_{1}+\alpha_{2} x_{2}\right)$. Unfortunately, Theorems 3.1 and 3.3 do not establish asymptotic independence of $X_{1}$ and $X_{2}$ for all parameters $\alpha_{1}, \alpha_{2} \in \mathbb{R}$ and $\omega \in(-1,1)$. Table 11 shows the range of parameter values for which asymptotic independence follows directly from these theorems, although we conjecture that it is true for all possible parameter values. By the definition of asymptotic independence it follows that the bivariate skew-normal distribution is in the maximum domain of attraction of (3.3) as long as $\alpha_{1}$ and $\alpha_{2}$ are as in Table 1 For $\omega \neq 0$ the asymptotic independence follows trivially from Theorems 3.1 and 3.3 If $\omega=0$ we have $\alpha_{1}=\bar{\alpha}_{1}$ and $\alpha_{2}=\bar{\alpha}_{2}$. Hence, for $\alpha_{1}, \alpha_{2}<0$ we can use Theorem 3.3 It is then enough to consider the case $\alpha_{2} \geq 0$. Taking i.i.d. $N(0,1)$ random variables $Z_{1}, Z_{2}$ we get, following the proof of Theorem 3.1 that $P\left(X_{1}>U_{1}(t), X_{2}>U_{2}(t)\right) \leq 2 P\left(Z_{1}>U_{1}(t)\right) P\left(Z_{2}>U_{2}(t)\right)$. Also 
Table 1

\begin{tabular}{c|c|c}
\hline $\boldsymbol{\omega}$ & $\boldsymbol{\alpha}_{\mathbf{1}}$ & $\boldsymbol{\alpha}_{\mathbf{2}}$ \\
\hline$\omega=0$ & $\alpha_{1} \in \mathbb{R}$ & $\alpha_{2} \in \mathbb{R}$ \\
\hline$\omega>0$ & $\alpha_{1} \geq 0$ & $\alpha_{2} \geq-\omega \alpha_{1}$ \\
& $\alpha_{1} \leq 0$ & $\alpha_{2} \geq-\frac{1}{\omega} \alpha_{1}$ \\
& $\alpha_{1}<0$ & $\alpha_{2}<0$ \\
\hline$\omega<0$ & $\alpha_{1} \geq 0$ & $-\omega \alpha_{1} \leq \alpha_{2} \leq-\frac{1}{\omega} \alpha_{1}$ \\
& $\alpha_{1}<0$ & $\alpha_{2}<0$ \\
\hline
\end{tabular}

Parameters $\alpha_{1}, \alpha_{2}$ and $\omega$ for which asymptotic independence holds for $S N_{2}\left(\alpha_{1}, \alpha_{2} ; \omega\right)$.

$P\left(X_{2}>U_{2}(t)\right) \geq P\left(Z_{2}>U_{2}(t)\right)$ since $\alpha_{2} \geq 0$. Hence, it follows that the limit in (3.1) is equal to 0 establishing the asymptotic independence in the $\omega=0$ case.

\section{Simulations}

The relevance of the results of Section 3 is determined by how many observations one has to have in order to make the asymptotic approximations acceptable. We first look at the rate of marginal convergence and then comment on the asymptotic independence using simulated skew-normal random vectors 2 .

One may expect a rather slow rate of convergence of the normalized maxima for the GSN distribution as is the case for the normal distribution which has a rate of $O\left(\frac{1}{\log n}\right)$ (cf. Hall $(1979)$ ), although computing the rate is still an open problem in the GSN case. Fig. 2a and 2b compare QQ-plots for 1,000 normalized maxima $\tilde{M}_{n}:=\left(M_{n}-b_{n}\right) / a_{n}$ with the block sizes $n=10^{3}$ and $n=10^{6}$, where $M_{n}:=\max _{1 \leq i \leq n} X_{i}, X_{i}$ 's are i.i.d. from $S N(\alpha)$ distribution and $a_{n}$ and $b_{n}$ are the normalizing constants from the extreme value theory. As can be seen from Fig. 2a, a random sample of size $10^{3}$ from a skew-normal distribution might not be sufficient to justify the use of the extreme value theory results. More convincing QQ-plots in Fig. 2b with $n=10^{6}$ confirm the statement of Proposition 2.4

To explore the rate at which components of the bivariate skew-normal random vectors approach independence, we first produced the so-called Chi-plots and $K$-plots to detect dependence in the simulated data; see Fig. 3 For details on

$\overline{2}$ The simulations were carried out with the statistical package $\mathbb{R}(2007)$ using library sn. 

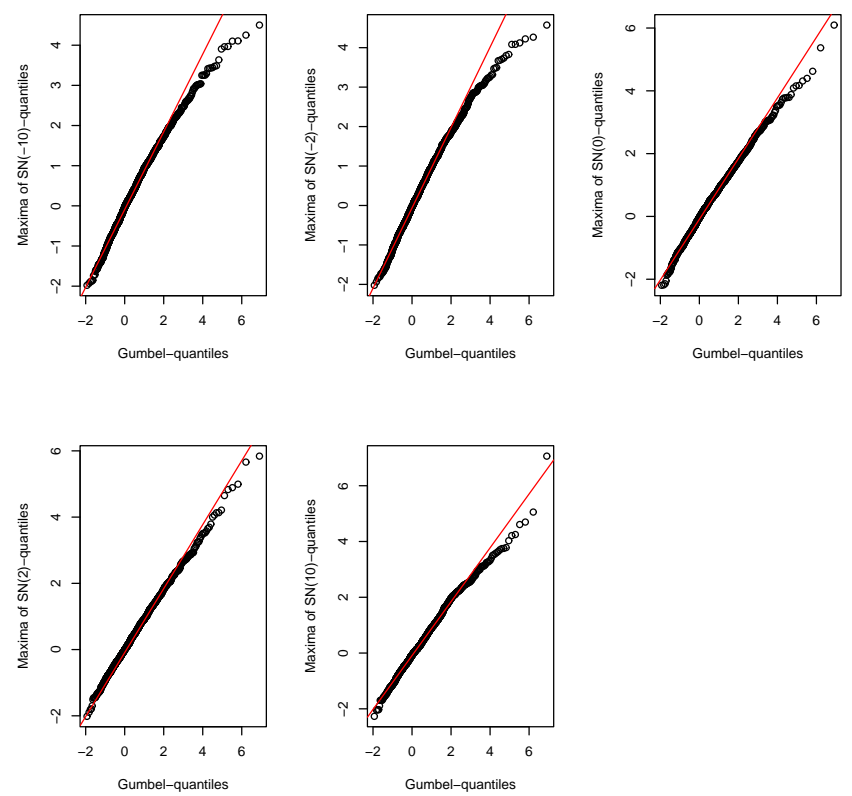

(a) $n=10^{3}$
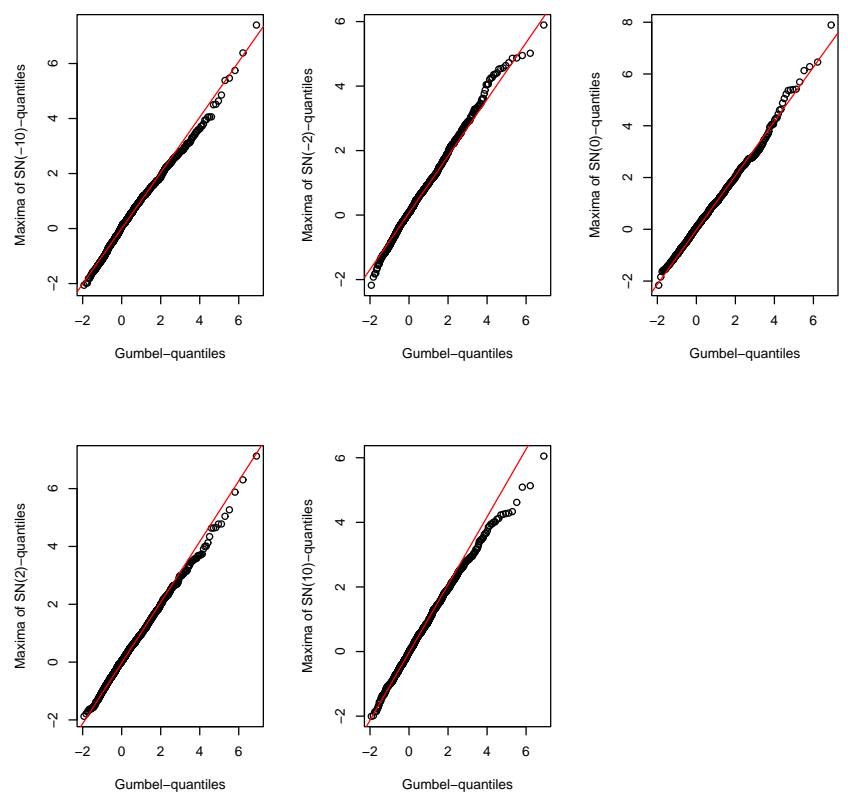

(b) $n=10^{6}$

Fig. 2. The QQ-plots for $\tilde{M}_{n}$, where $X$ follows a skew-normal distribution with $\alpha=-10,-2,0,2,10$.

how to construct these plots and justifications, see Genest and Favre (2007). In the Chi-plot of pairs $\left(\lambda_{i}, \chi_{i}\right)$, the values of $\chi_{i}$ away from zero indicate departures from the hypothesis of independence. The horizontal dashed bounds are drawn at the levels $\pm c_{p} / \sqrt{k}$ with $c_{p}=2.18$ so that approximately $99 \%$ of the pairs $\left(\lambda_{i}, \chi_{i}\right)$ lie within these bounds (cf. Genest and Favre (2007)). 


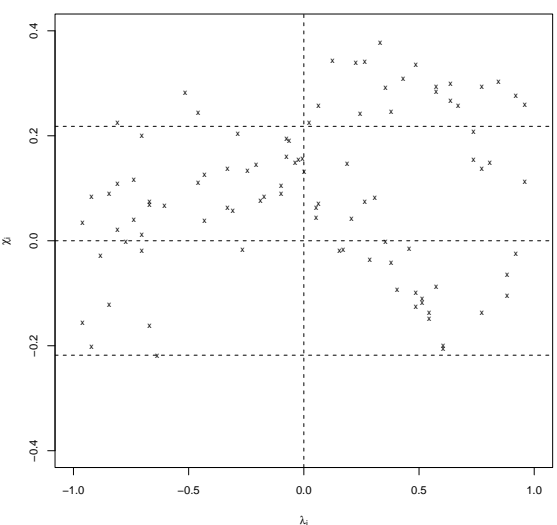

(a) Chi-plot for $\mathbf{X}$

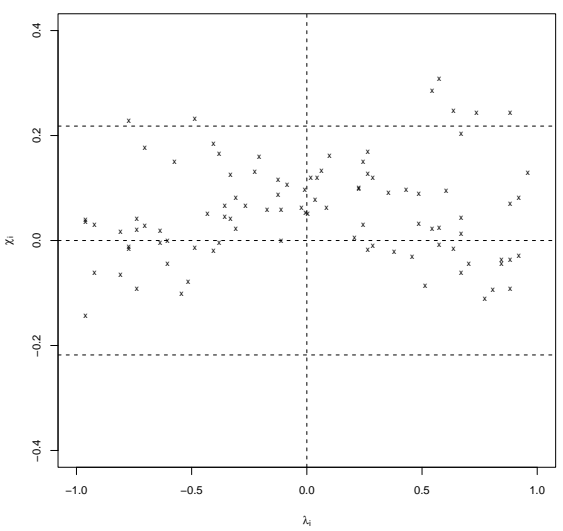

(c) Chi-plot for $\mathbf{M}_{10^{3}}$

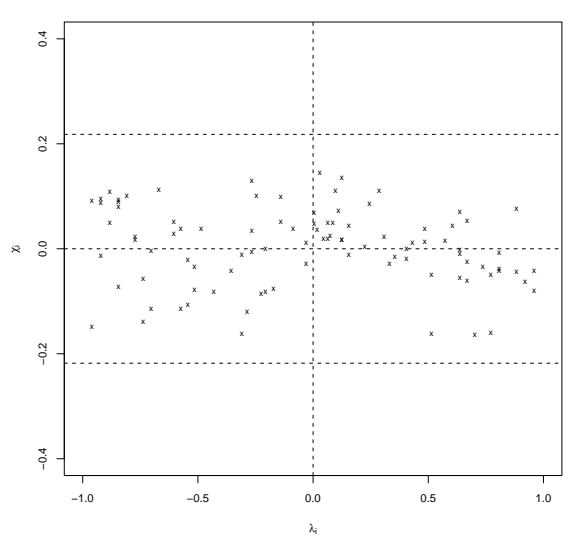

(e) Chi-plot for $\mathbf{M}_{10^{6}}$

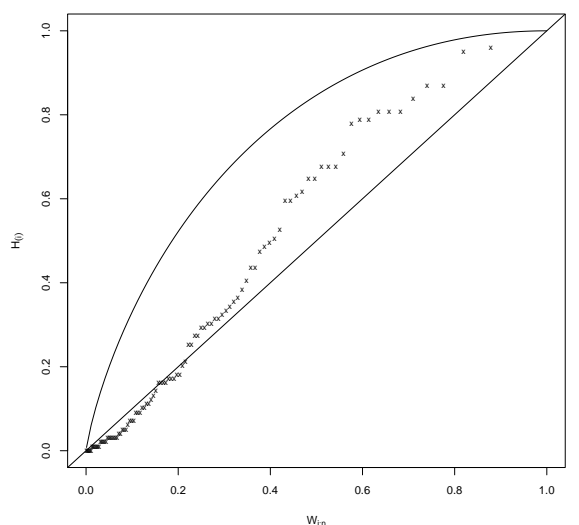

(b) K-plot for $\mathbf{X}$

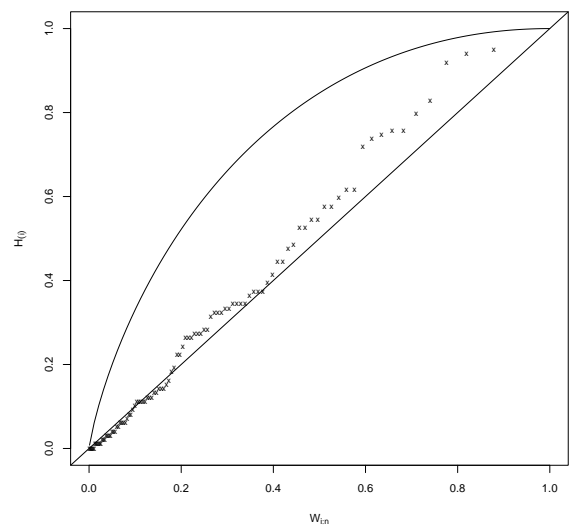

(d) K-plot for $\mathbf{M}_{10^{3}}$

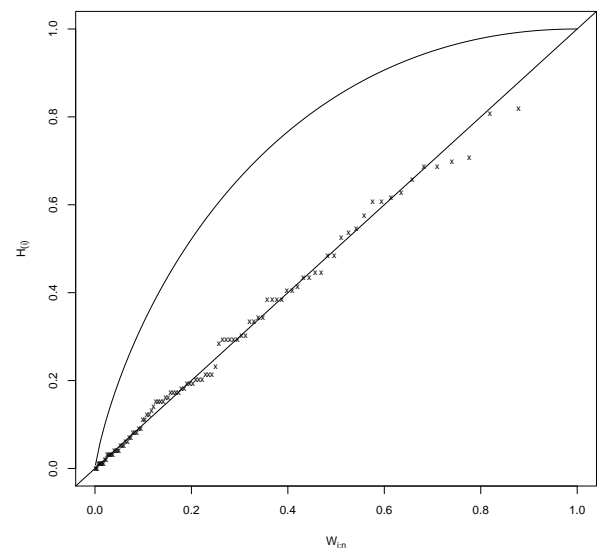

(f) K-plot for $\mathbf{M}_{10^{6}}$

Fig. 3. Chi-plots and K-plots for simulated random samples of size 100 for $\mathbf{X} \sim S N_{2}\left(\alpha_{1}, \alpha_{2} ; \omega\right)(\mathrm{a})$-(b) and the corresponding coordinatewise maxima $\mathbf{M}_{n}$ with block sizes $n=10^{3}$ (c)-(d) and $n=10^{6}$ (e)-(f). The parameter values are $\alpha_{1}=2$, $\alpha_{2}=3$ and $\omega=0.6$. 


\begin{tabular}{|c|c|c|c|c|c|c|}
\hline \multicolumn{7}{|c|}{$\omega=0.6$} \\
\hline & \multicolumn{2}{|c|}{$\alpha_{1}=2, \alpha_{2}=3$} & \multicolumn{2}{|c|}{$\alpha_{1}=2, \alpha_{2}=-3$} & \multicolumn{2}{|c|}{$\alpha_{1}=-2, \alpha_{2}=3$} \\
\hline$n$ & $p_{\rho}$ & $p_{\tau}$ & $p_{\rho}$ & $p_{\tau}$ & $p_{\rho}$ & $p_{\tau}$ \\
\hline $10^{6}$ & 1 & 1 & 0.78 & 0.78 & 0.98 & 0.98 \\
\hline $10^{4}$ & 0.97 & 0.96 & 0.54 & 0.55 & 0.98 & 0.98 \\
\hline $10^{3}$ & 0.97 & 0.97 & 0.18 & 0.18 & 0.89 & 0.89 \\
\hline $10^{2}$ & 0.87 & 0.86 & 0.18 & 0.18 & 0.50 & 0.48 \\
\hline \multicolumn{7}{|c|}{$\omega=0.1$} \\
\hline $10^{6}$ & 1 & 0.99 & 1 & 0.99 & 0.99 & 0.99 \\
\hline $10^{4}$ & 1 & 1 & 1 & 1 & 0.99 & 0.99 \\
\hline $10^{3}$ & 1 & 1 & 0.96 & 0.97 & 0.99 & 0.99 \\
\hline $10^{2}$ & 1 & 1 & 0.79 & 0.76 & 0.99 & 0.99 \\
\hline \multicolumn{7}{|c|}{$\omega=0$} \\
\hline $10^{6}$ & 0.99 & 0.98 & 1 & 1 & 1 & 1 \\
\hline $10^{4}$ & 1 & 1 & 1 & 1 & 0.98 & 0.98 \\
\hline $10^{3}$ & 0.99 & 0.99 & 1 & 1 & 0.99 & 0.99 \\
\hline $10^{2}$ & 0.99 & 0.98 & 1 & 1 & 0.99 & 0.99 \\
\hline \multicolumn{7}{|c|}{$\omega=-0.1$} \\
\hline $10^{6}$ & 1 & 1 & 0.98 & 0.98 & 1 & 1 \\
\hline $10^{4}$ & 1 & 1 & 0.96 & 0.96 & 0.97 & 0.98 \\
\hline $10^{3}$ & 0.99 & 1 & 0.99 & 0.99 & 0.98 & 0.98 \\
\hline $10^{2}$ & 1 & 1 & 0.99 & 0.99 & 0.99 & 0.99 \\
\hline \multicolumn{7}{|c|}{$\omega=-0.6$} \\
\hline $10^{6}$ & 0.98 & 0.98 & 1 & 1 & 1 & 1 \\
\hline $10^{4}$ & 1 & 1 & 0.97 & 0.97 & 1 & 1 \\
\hline $10^{3}$ & 0.99 & 0.99 & 1 & 1 & 0.99 & 0.99 \\
\hline $10^{2}$ & 1 & 1 & 1 & 1 & 0.99 & 0.99 \\
\hline
\end{tabular}

Table 2

Proportions of the tests based on Spearman's rho $\left(p_{\rho}\right)$ and Kendall's tau $\left(p_{\tau}\right)$ for which there was not enough evidence to reject the null hypothesis of independence of the coordinatewise maxima at level $\alpha=1 \%$. Simulated data: 100 block maxima $\mathbf{M}_{n}$ from $S N_{2}\left(\alpha_{1}, \alpha_{2} ; \omega\right)$ distribution; each test was repeated 100 times. 
The $45^{\circ}$-line on the K-plot corresponds to the case of independence, and the superimposed curve corresponds to the case of perfect positive dependence. As can be seen from Fig. 3a and 3b, the components of the $S N_{2}(2,3 ; 0.6)$ random vector exhibit positive dependence, which eventually disappears for the maxima as the block size $n$ becomes large; see Fig. 3c- $3 \mathrm{f}$.

Using Spearman's rho and Kendall's tau, we test the hypothesis of independence of coordinatewise maxima from an $S N_{2}\left(\alpha_{1}, \alpha_{2} ; \omega\right)$ distribution. Again the reader is referred to Genest and Favre (2007) and Genest and Verret (2005) for details on rank-based tests of independence. The tests are based on 100 blocks of coordinatewise maxima with block size $n$, and each test is repeated 100 times. In Table 2 we report proportions of the tests which could not reject the null hypothesis of independence at approximately level $\alpha=1 \% ; p_{\rho}$ and $p_{\tau}$ denote the proportion of the tests based on Spearman's rho and Kendall's tau, respectively, with the $\mathrm{P}$-values exceeding 0.01 . The values highlighted in bold correspond to the choice of parameters within the range specified in Table 1 for which we have an analytical proof of asymptotic independence. These results indicate that even relatively small block sizes such as $n=100$ and $n=1000$ are sufficient for the convergence of the maximal components to being independent. The rest of the values support our conjecture that in fact asymptotic independence holds for all possible parameter values. Low proportions of insignificant tests corresponding to $\alpha_{1}=2, \alpha_{2}=-3, \omega=0.6$ might be due to slower rates of convergence. A further analytical investigation of the asymptotic independence property is required.

Acknowledgments. The authors are thankful to Matthias Degen, Paul Embrechts, Dominik Lambrigger and Johanna Neslehova for several useful discussions related to the paper, and to the anonymous referee for his/her comments which helped to improve the presentation of the paper.

\section{References}

Azzalini, A., Capitanio, A., 1999. Statistical applications of the multivariate skew normal distribution. Journal of the Royal Statistical Society. Series B (Statistical Methodology) 61:579-602.

Azzalini, A., Dalla Valle, A., 1996. The multivariate skew-normal distribution. Biometrika 83:715-726.

Balkema, A. A., Embrechts, P., 2007. High Risk Scenarios and Extremes. A Geometric Approach. Zurich Lecture Notes, European Mathematical Society.

Chang, S. M., Genton, M. G., 2007. Extreme value distributions for the skewsymmetric family of distributions. Communications in Statistics-Theory and Methods 36:1705-1717. 
Embrechts, P., Klüppelberg, C., Mikosch, T., 1997. Modelling Extremal Events for Insurance and Finance. Springer, Berlin.

Genest, C., Favre, A., 2007. Everything you always wanted to know about copula modeling but were afraid to ask. Journal of Hydrologic Engineering $12: 347-368$.

Genest, C., Verret, F., 2005. Locally most powerful rank tests of independence for copula models. Journal of Nonparametric Statistics 17:521-539.

Genton, M. G., 2004. Skew-elliptical Distributions and Their Applications : A Journey Beyond Normality. Chapman \& Hall, Boca Raton.

de Haan, L., Ferreira, A., 2006. Extreme Value Theory. Springer, New York.

P. Hall (1979): On the rate of convergence of normal extremes. Journal of Applied Probability 16:433-439.

Hill, M., Dixon, W., 1982. Robustness in real life: a study of clinical laboratory data. Biometrics 38:377-396.

Lane, M. N., 2000. Pricing risk transfer transactions. Astin Bulletin 30:259293.

Ma, Y., Genton, M. G., 2004. Flexible class of skew-symmetric distributions. Scandinavian Journal of Statistics 31:459-468.

McNeil, A. J., Frey, R., Embrechts, P., 2005. Quantitative Risk Management. Concepts, Techniques and Tools. Princeton University Press, Princeton, NJ.

R, 2007. R: A Language and Environment for Statistical Computing.R Foundation for Statistical Computing, Vienna, Austria.

Resnick, S., 1987. Extreme Values, Regular Variation, and Point Processes. Springer, New York.

Sibuya, M., 1960. Bivariate extreme statistics. Annals of the Institute of Statistical Mathematics 11:195-210. 